\title{
Lateral-force measurements in dynamic force microscopy
}

\author{
O. Pfeiffer, R. Bennewitz, ${ }^{*}$ A. Baratoff, and E. Meyer \\ Department of Physics, University of Basel, Klingelbergstrasse 82, 4058 Basel, Switzerland \\ P. Grütter \\ Physics Department, McGill University, 3600 University Street, Montréal, Canada H3A 2T8
}

(Received 21 December 2001; published 8 April 2002)

\begin{abstract}
Lateral forces between the tip of a force microscope and atomic-scale features on the surface of a sample can be accurately measured in a noncontact mode. Feedback-controlled excitation of the torsional eigenmode of a rectangular cantilever beam forces the tip to oscillate parallel to the surface. Forces of the order of $0.05 \mathrm{nN}$ have been detected when the tip approaches a step or a sulphur impurity. The method can also be used to study the energy dissipation in the range where a tip-sample contact is formed.
\end{abstract}

DOI: 10.1103/PhysRevB.65.161403

PACS number(s): 68.37.Ps, 07.79.Lh

\section{INTRODUCTION}

Lateral forces in the nanonewton regime govern the activated or induced movement of molecular-scale objects along surfaces. Their measurement is an important issue in manipulation experiments ${ }^{1}$ as well as in force microscopy per se. Studies of sharp tips sliding in gentle contact over sample surfaces have already contributed to the understanding of the microscopic origins of friction forces. ${ }^{2}$ However, the lateral resolution of force microscopy in the contact mode is limited by a minimum contact area containing several atoms due to adhesion between tip and sample. This problem has been overcome in noncontact dynamic force microscopy. True atomic resolution is obtained thanks to short-range interaction between a sample and a tip, which is forced to oscillate normal to the surface. ${ }^{3}$

Jarvis et al. developed a special cantilever layout for simultaneous control of tip-sample distance and lateral tip oscillations in order to sense lateral interactions between tip and sample when approaching the surface. ${ }^{4}$ In this article, we report experiments using the torsional oscillation of a rectangular cantilever beam. For oscillation amplitudes small compared to the height of the tip, its apex essentially moves parallel to the surface [see Fig. 1(a)]. After an introduction of the experimental technique we demonstrate that lateral forces between objects on the surface and the tip can be measured via the induced change of the frequency of the fundamental torsional eigenmode. The tip-sample distance in these experiments is controlled by keeping constant the time-averaged tunneling current. Finally, we discuss the change of the oscillatory behavior when the tip is further approached towards the surface and a tip-sample contact is formed.

\section{EXPERIMENTAL TECHNIQUE}

Our instrument is a home-built combined tunneling and force microscope operating at room temperature in ultrahigh vacuum (UHV). We use commercially available rectangular conductive silicon cantilevers with an integrated tip. The deformation of the cantilever is detected via the deflection of a light beam reflected from the rear side of the cantilever. As in simultaneous load and friction experiments, a four-quadrant photodiode is employed to detect both bending and torsional deflections. In order to prevent the tip from jumping into contact with the surface due to the strong force gradients likely to develop at tunneling distances, relatively stiff cantilevers with a bending force constant $k_{n}>25 \mathrm{~N} / \mathrm{m}$ are required. The eigenfrequency $f_{0}$ of the fundamental torsional mode of our cantilevers is typically between $1 \mathrm{MHz}$ and 3 $\mathrm{MHz} ;{ }^{5}$ the quality factor in UHV being between 20000 and 40000 , and the torsional force constant being about $k_{t}$ $=3000 \mathrm{~N} / \mathrm{m}^{6}{ }^{6}$ The detection of beam deflection at these frequencies in ultrahigh vacuum necessitated some improvements in the signal amplification. In our instrument, the position of the reflected light beam is sensed by a negatively biased low-capacitance photodiode. The currents from the four quadrants are converted to voltages by fast operation amplifiers which are positioned a few millimeters from the photodiode. To circumvent heating problems in vacuum, the amplifiers are operated at low voltage. The photodiode and the current-to-voltage converter are mounted on a UHVcompatible sapphire circuit board. Directly outside the vacuum chamber the voltage signal is again amplified to compensate the capacitive load of the output cable. Actual eigenfrequencies of the cantilever oscillation up to $3 \mathrm{MHz}$ are detected by a digital phase-locked loop with a resolution of $10 \mathrm{mHz} .^{7}$ A sinusoidal signal with the same frequency is used to excite the torsional oscillation by means of a piezoactuator. Although the actuator mainly shakes the cantilever holder in the normal direction, the high quality factor and the tuning to the actual eigenfrequency ensure that only the torsional oscillation is effectively excited. The amplitude of ex-
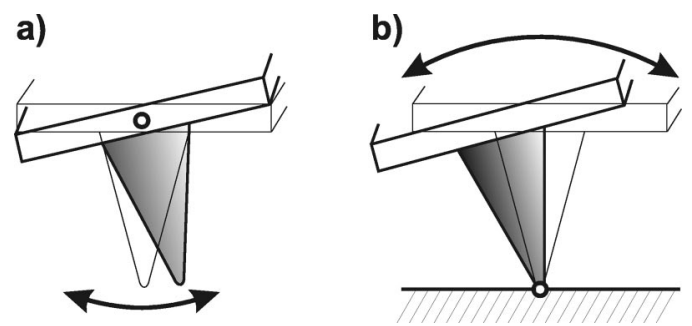

FIG. 1. Torsional modes of cantilever oscillation. (a) Free oscillation, the tip is oscillating parallel to the surface. (b) Torsional oscillation with the tip-sample contact as fixed point. 
citation is controlled so as to always maintain a constant amplitude $A$ of the tip oscillation.

The (100) surface of a $\mathrm{Cu}$ single crystal has been cleaned by repeated cycles of argon ion sputtering and annealing. After this preparation, the surface exhibits atomically flat terraces of typically $100 \mathrm{~nm}$ width, separated by monatomic steps. Traces of sulphur detected in Auger electron spectra appear as single impurities in tunneling microscopy images.

\section{LATERAL FORCE MEASUREMENTS}

In the following section, we describe how lateral forces between tip and features on the surface can be measured via the oscillation frequency. We record shifts of the frequency of the lateral oscillation while scanning the tip at constant tunneling current. Note that normal forces between tip and surface do not shift the frequency. However, lateral forces between any object on the surface and the tip are detected. In Fig. 2(a), a map of the frequency shift recorded on a $\mathrm{Cu}(100)$ surface is presented. Several sulphur impurities and a monatomic step running through the middle of the frame appear twice, once at each of the two turning points of the tip oscillation. Half of the distance between the members of each pair of features corresponds to an amplitude $A=2 \mathrm{~nm}$ of the lateral tip oscillation: When the oscillating tip approaches from one or the other side, the force between tip and object is sensed particularly at the closest turning point of the oscillation. Profiles of the topography and the frequency shift along the white line are plotted in Fig. 2(b). When the tip approaches the step from the left (lower terrace), a negative frequency shift occurs, while an increase is detected when the step is approached from the right (upper terrace). At the sulphur impurity, a decrease followed by an increase is observed symmetrically upon approaching from either side. Note that the sulphur impurity is not detected as topographic effect, but via its lateral force. This demonstrates that the tip oscillates at a safe distance and that our technique is extremely sensitive. We next explain how these frequency shifts can be converted into lateral forces and discuss the specific variations observed near the step and the impurity.

The net force acting along the direction of the tip oscillation manifests itself as a shift of the mechanical eigenfrequency. Generally, the frequency is lowered when the interaction reduces the effective spring constant. This situation is found in standard noncontact force microscopy when the tip oscillates perpendicular to a surface and electrostatic, van der Waals, or chemical bonding forces become more attractive with decreasing distance to the surface. ${ }^{8}$ On the other hand, repulsive forces are expected to increase the eigenfrequency of the oscillation. Normally, the approaching tip first senses attractive forces and then a mix of attractive forces acting on the tip body and of repulsive forces between tip apex and sample surface. The same considerations can be applied to lateral forces acting on the tip when oscillating parallel to the surface. In our example, the negative frequency shift upon approach of the surface step from the lower terrace indicates an attractive lateral force towards the step.

A numerical procedure to convert frequency vs distance curves into force vs distance curves has been proposed by
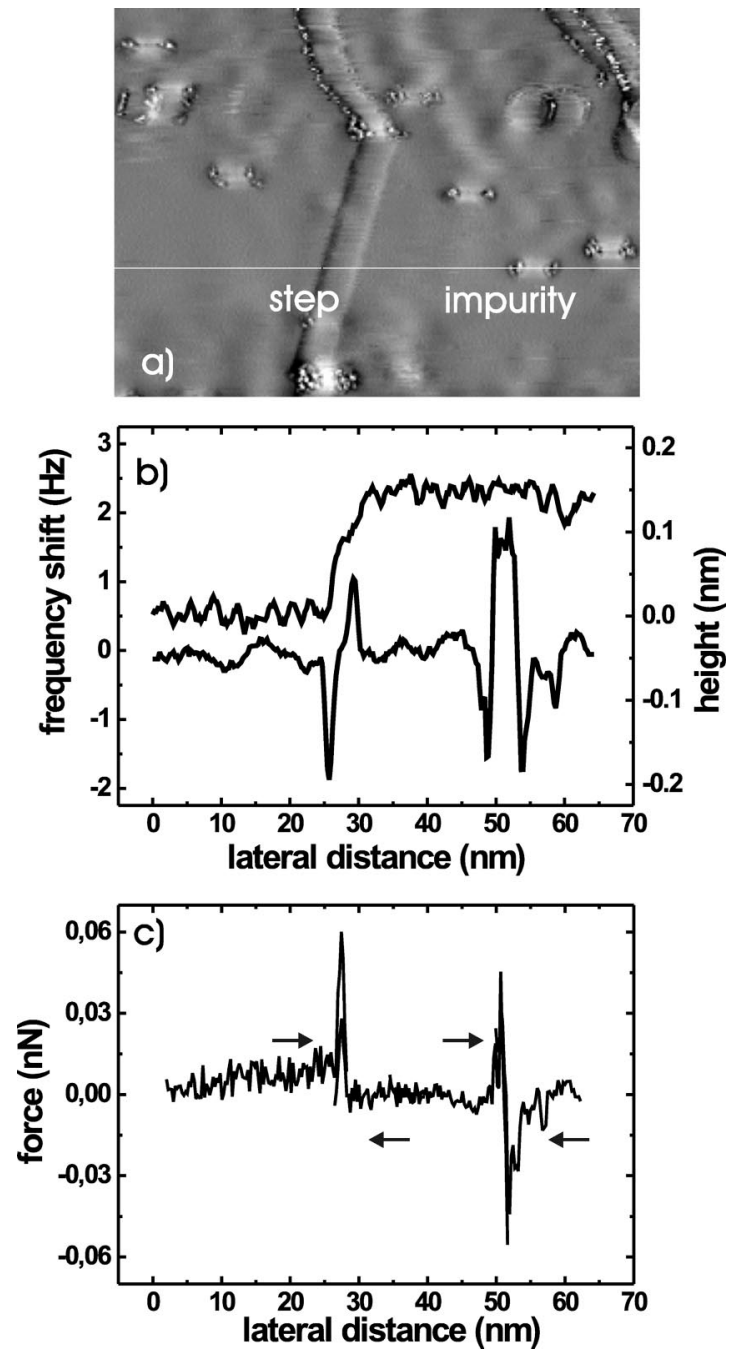

FIG. 2. (a) Frequency shift map of the lateral oscillation recorded while scanning at constant tunneling current on a $\mathrm{Cu}(100)$ surface (full range $3.5 \mathrm{~Hz}$ ). A monatomic step running from top to bottom and several sulphur impurities appear twofold due to the lateral tip oscillation $\left(f_{0}=1346929 \mathrm{~Hz}, U_{\text {sample }}=0.8 \mathrm{~V}, I_{t}\right.$ $=10 \mathrm{pA}$ ). An oscillation amplitude of $A=2 \mathrm{~nm}$ can be deduced. (b) Cross-section of topography (upper curve) and frequency shift (lower curve) along the white bar. (c) Lateral force $F(x)$ computed by the inversion scheme from four lateral approach curves (directions indicated by arrows). Positive and negative values refer to the horizontal axis running from left to right.

Giessibl. ${ }^{9}$ We have adapted his inversion scheme with some modifications. The underlying expression

$$
\Delta f(x)=-\frac{f_{0}}{\pi k_{t} A} \int_{-1}^{1} \frac{F(x+A u) u}{\sqrt{1-u^{2}}} d u
$$

relating the frequency shift $\Delta f$ to the lateral force $F(x)$ over the oscillation cycle is valid if the relative shift $\Delta f / f_{0}$ is small, which is certainly the case. The basic idea is to discretize the integral in Eq. (1) and to treat the equations between $\Delta f$ and $F$ at the measurement points as a linear system. If a feature of interest is approached from a range where $\Delta f$ vanishes, the resulting triangular matrix can be inverted 


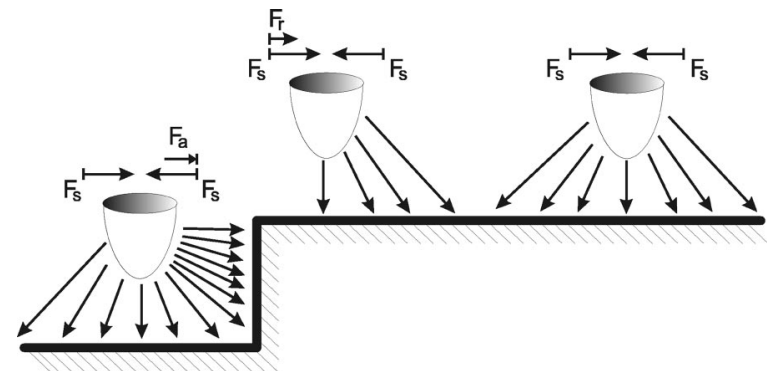

FIG. 3. Schematic illustration of the changing interaction area and of the resulting lateral forces near a step. Because force $F_{a}$ lowers the restoring spring force $F_{s}$, the resonance frequency is lowered when the right turning point approaches the step. Conversely, a positive frequency shift is induced by force $F_{r}$ on the other side.

by substitution. The square root singularities at the turning points $x \pm A$ can be handled by analytic integration of $u / \sqrt{1-u^{2}}$ between the meshpoints. It is important to realize that the sign of $F(x)$ depends on the chosen direction of the $x$ axis, whereas attraction or repulsion refer to the location of any particular feature.

The result of the inversion is plotted in Fig. 2(c). The curve is composed of four pieces, the approach from the left and from the right to the step and to the impurity, as indicated by arrows. Note that the resulting force curves almost coincide at the inferred locations of each feature. In the noncontact range the tip is attracted towards the impurity. Thus, the lateral force switches its sign as the tip passes over it. This happens twice: When the right and then the left turning point cross the impurity. This explains the observed variation of the frequency shift. The situation at the step is complicated by the stairlike change in the tip height. However, the observed attraction towards the step on the lower terrace and the repulsion away from the step on the upper terrace can be understood in terms of increased or decreased interaction areas, respectively, as illustrated in Fig. 3. A similar variation can also be observed for the normal forces. ${ }^{10}$

\section{CONTACT FORMATION}

We now discuss changes of the lateral oscillation which occur when the tip is further approached towards the surface. As seen in Fig. 4b, a rapid increase of the power required to maintain a constant tip oscillation amplitude and a positive frequency shift are found. A similar increase of the excitation power is observed in the case of a bending oscillation normal to the surface [see Fig. 4(a) and Ref. 11]. Note that in Fig. 4(b) the onset of the simultaneously recorded damping and positive frequency shift are sharp and essentially coincide. We suggest that these changes indicate the formation of a tip-sample contact. We have assigned the zero of the distance axis to the point where the tunneling current amplifier saturated at $10 \mathrm{nA}$. Judging from previous studies on copper surfaces, ${ }^{12}$ this zero is within a few $\AA$ of the point of mechanical contact, essentially because the tunneling current decays on that scale.

Two recent studies report on the use of the torsional
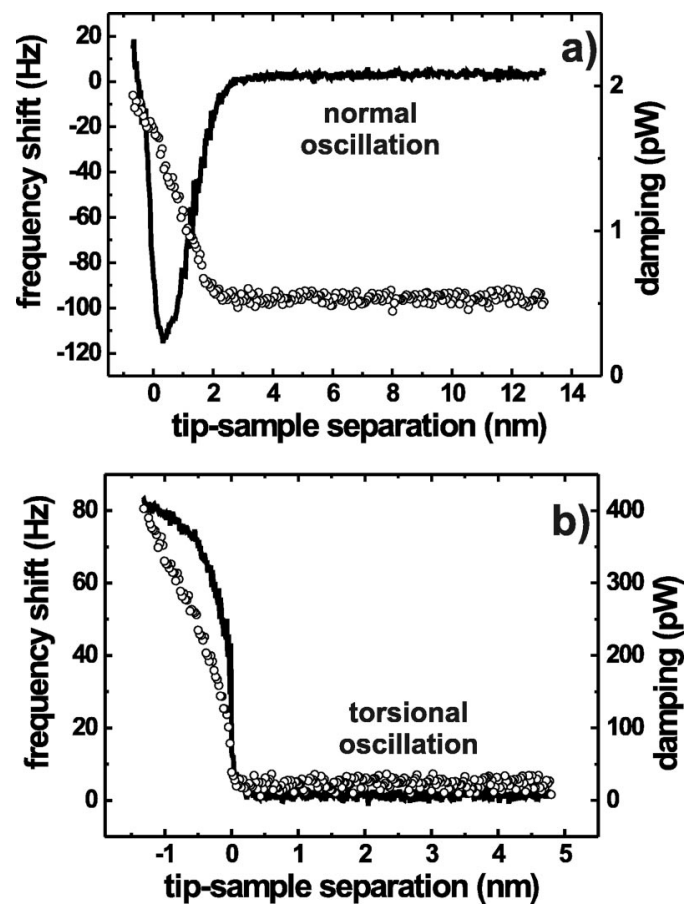

FIG. 4. Frequency (solid line) and damping (open circles) vs sample displacement curves. (a) Normal oscillation $\left(f_{0}\right.$ $=154905 \mathrm{~Hz}, A=30 \mathrm{~nm}, Q=21000, k_{n}=25.7 \mathrm{~N} / \mathrm{m}$ ). (b) Lateral oscillation parallel to the surface $\left(f_{0}=1270283 \mathrm{~Hz}, A\right.$ $\left.=6 \mathrm{~nm}, Q=19000, k_{t}=2925 \mathrm{~N} / \mathrm{m}\right)$. The zeros of the two horizontal scales do not coincide and are uncertain within a few $\AA$ (see text).

eigenmode for measuring the dynamic properties of the tipsample contact. ${ }^{13,14}$ In those studies, the tip is in firm contact with the sample so that a modified torsional mode with the contact-point fixed is excited [see Fig. 1(b)]. The eigenfrequency and the quality factor of this oscillation depend on the lateral stiffness of the contact, which itself increases with the applied normal load. The authors report that the eigenfrequency with the tip in contact is slightly higher than that of the freely oscillating cantilever, while the quality factor decreases with increasing lateral stiffness. ${ }^{14}$ Both observations are consistent with the trends observed in Fig. 4(b).

In dynamic lateral force experiments one has to make sure that the oscillation is indeed parallel to the surface. When the surface is tilted, we find small negative frequency shifts, which can be explained by oscillation components normal to the surface, in line with Fig. 4(a) and Ref. 4.

The idea to relate additional damping of the tip oscillation to dissipative tip-sample interactions has recently attracted much attention. ${ }^{15}$ It has been demonstrated that in conventional dynamic force microscopy power loss variations as low as $100 \mathrm{meV}$ per oscillation cycle can be detected with atomic resolution. ${ }^{12}$ In our example, the damping vs distance curve for the normal oscillation reaches a maximum of about $2 \mathrm{pW}$, corresponding to $80 \mathrm{eV}$ per oscillation cycle, where the frequency shift becomes positive [see Fig. 4(a)]. In the case of the lateral oscillation, a maximal power loss of 400 $\mathrm{pW}$ is detected after contact formation, corresponding to about $2000 \mathrm{eV}$ per oscillation cycle, a comparatively high 
energy loss. However, one has to keep in mind that the contact acts as a hinge in this oscillation mode. Forces of the order of the restoring force $k_{t} A=18 \mu \mathrm{N}$ act on the contact with a frequency of $1.3 \mathrm{MHz}$ and may cause such energy dissipation by exciting shear waves. The assumption of contact formation is further supported by the strong adhesion hysteresis observed when the tip is retracted.

\section{SUMMARY}

In summary, we have demonstrated the measurement of lateral forces in dynamic force microscopy by exciting the torsional oscillation mode of a conducting silicon cantilever beam. By using the tunneling current to control the distance to a metallic sample in the non-contact regime, attraction and repulsion at a monatomic step have been quantified, as well as the force between an impurity and the tip. Lateral forces play a crucial role in manipulation experiments on the molecular level. ${ }^{16}$ Our method is more than sensitive enough to monitor the onset of the lateral force required for the ma- nipulation of adsorbates on the surface. Besides the measurement of lateral forces in the noncontact mode, the formation of a tip-sample contact can be studied by our technique. When the laterally oscillating tip is approached, contact formation transforms the nearly free torsional oscillation [see Fig. 1(a)] of the cantilever into a different mode with the tip-sample contact acting as fixed point [see Fig. 1(b)]. We have detected concomitant steep increases in dissipation and torsional eigenfrequency upon formation of the contact, which are consistent with previous measurements in the fixed-point regime.

\section{ACKNOWLEDGMENTS}

We would like to thank E. Gnecco and R. Hoffmann for fruitful discussions. This work was supported by the Swiss National Science Foundation, the "Kommission zur Förderung von Technologie und Innovation" and the National Center of Competence in Research on Nanoscale Science.
*Electronic address: roland.bennewitz@unibas.ch

${ }^{1}$ J. Stroscio and D. Eigler, Science 254, 319 (1991).

${ }^{2}$ C. Mate, G. McClelland, R. Erlandsson, and S. Chiang, Phys. Rev. Lett. 59, 1942 (1987).

${ }^{3}$ Proceedings of the Third International Workshop on Noncontact Atomic Force Microscopy, edited by U. Schwarz, H. Hölscher, and R. Wiesendanger, Vol. S72 of Applied Physics A (Springer, Heidelberg, 2001).

${ }^{4}$ S. Jarvis, H. Yamada, K. Kobayashi, A. Toda, and H. Tokumoto, Appl. Surf. Sci. 157, 314 (2000).

${ }^{5}$ U. Rabe, K. Janser, and W. Arnold, Rev. Sci. Instrum. 67, 3281 (1996)

${ }^{6}$ O. Pfeiffer, C. Loppacher, C. Wattinger, M. Bammerlin, U. Gysin, M. Guggisberg, R. Bennewitz, E. Meyer, and H.-J. Güntherodt, Appl. Surf. Sci. 157, 337 (2000).

${ }^{7}$ C. Loppacher, M. Bammerlin, F. Battiston, M. Guggisberg, D. Müller, H. Hidber, R. Lüthi, E. Meyer, and H.-J. Güntherodt, Appl. Phys. A 66, 215 (1998).
${ }^{8}$ F. Giessibl, Phys. Rev. B 56, 16010 (1997).

${ }^{9}$ F. Giessibl, Appl. Phys. Lett. 78, 123 (2001).

${ }^{10}$ M. Guggisberg, M. Bammerlin, A. Baratoff, R. Lüthi, C. Loppacher, F. Battiston, J. Lü, R. Bennewitz, E. Meyer, and H.-J. Güntherodt, Surf. Sci. 461, 255 (2000).

${ }^{11}$ C. Loppacher, R. Bennewitz, O. Pfeiffer, M. Guggisberg, M. Bammerlin, S. Schär, V. Barwich, A. Baratoff, and E. Meyer, Phys. Rev. B 62, 13674 (2000).

${ }^{12}$ C. Loppacher, M. Bammerlin, M. Guggisberg, S. Schär, R. Bennewitz, A. Baratoff, E. Meyer, and H.-J. Güntherodt, Phys. Rev. B 62, 16944 (2000).

${ }^{13}$ K. Yamanaka, A. Naguchi, T. Tsuji, T. Koike, and T. Goto, Surf. Interface Anal. 27, 600 (1999).

${ }^{14}$ T. Drobek, R. Stark, and W. Heckl, Phys. Rev. B 64, 045401 (2001).

${ }^{15}$ M. Gauthier and M. Tsukada, Phys. Rev. B 60, 11716 (1999).

${ }^{16}$ T. Jung, R. Schlittler, J. Gimzewski, H. Tamg, and C. Joachim, Science 271, 181 (1996). 